\title{
Application of the Virtual Fields Method to a relaxation behaviour of rubbers
}

\author{
Sung-ho Yoon ${ }^{1, *}$ and Clive R Siviour ${ }^{1}$
}

${ }^{1}$ Department of Engineering Science, University of Oxford, UK

*sung-ho.yoon@eng.ox.ac.uk

\section{KEYWORD}

Rubber, Viscoelasticity, Mechanical characterization, Virtual fields method

\begin{abstract}
This paper presents the application of the Virtual Fields Method (VFM) for the characterization of viscoelastic behaviour of rubbers. The relaxation behaviour of the rubbers following a dynamic loading event is characterised using the dynamic VFM in which full-field (two dimensional) strain and acceleration data, obtained from high-speed imaging, are analysed by the principle of virtual work without traction force data, instead using the acceleration fields in the specimen to provide stress information. Two (silicone and nitrile) rubbers were tested in tension using a drop-weight apparatus. It is assumed that the dynamic behaviour is described by the combination of hyperelastic and Prony series models. A VFM based procedure is designed and used to produce the identification of the modulus term of a hyperelastic model and the Prony series parameters within a time scale determined by two experimental factors: imaging speed and loading duration. Then, the time range of the data is extended using experiments at different temperatures combined with the time-temperature superposition principle. Prior to these experimental analyses, finite element simulations were performed to validate the application of the proposed VFM analysis. Therefore, for the first time, it has been possible to identify relaxation behaviour of a material following dynamic loading, using a technique that can be applied to both small and large deformations.
\end{abstract}

\section{INTRODUCTION}

The loading rate dependency of the response of rubbers to mechanical deformation is mainly due to time-dependent motion of the polymer chains when topologically constrained by a molecular network. At a high enough global deformation rate, the free chain is affinely strained with the network. If the deformation is applied slowly, there is sufficient time for the chain to move to a more relaxed configuration (Bergström and Boyce, 1998). Many rubbers are highly viscoelastic; hence the stress-strain relationship is clearly different even for modest changes in strain rate. Much research has therefore been conducted characterizing distinctive mechanical behaviours of rubber at different loading rates, often using the split Hopkinson compression bar (SHPB) for dynamic experiments (Chen, 2016; Gray and Blumenthal, 2000; Siviour and Jordan, 2016). There are two significant challenges in performing these experiments: in order to use the established analysis, the specimen size must be small so that the specimen can reach a state of stress equilibrium on the time scale of the dynamic experiment, and the forces supported by the specimen are often small, requiring sensitive instrumentation. To address this issue, the conventional SHPB has been modified by using: polymeric bars (Bacon, 1998; Harrigan et al., 2014), hollow metallic bars (Chen et al., 1999), piezoelectric sensors (Chen et al., 2000; Kendall et al., 2014) and pulse shaping (Song and Chen, 2003).

A number of authors have conducted dynamic tensile tests on elastomers with some modifications to the traditional split Hopkinson tension bar (SHTB), which was first developed for experiments on metals (Harding et al., 1960). Cheng and Chen (2003) used a short specimen and pulse shaping technique for a SHTB test on EPDM rubber at a strain rate of about $3000 \mathrm{~s}^{-1}$. A similar experiment was conducted on polyurethane using a traditional SHTB in combination with a

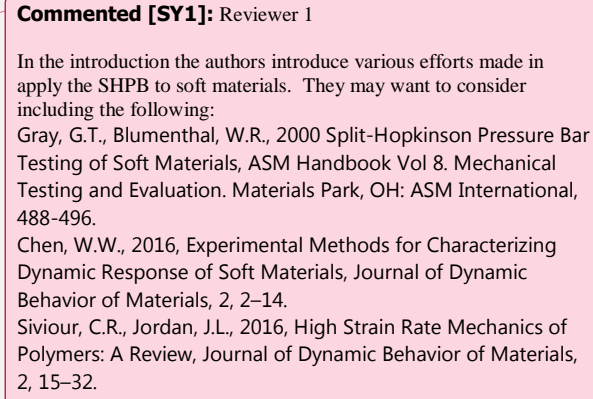


pendulum striker (Kanyanta and Ivankovic, 2010). Apart from the conventional Hopkinson bar technique, Roland et al. (2007) developed a special drop-weight test apparatus in which a tensile

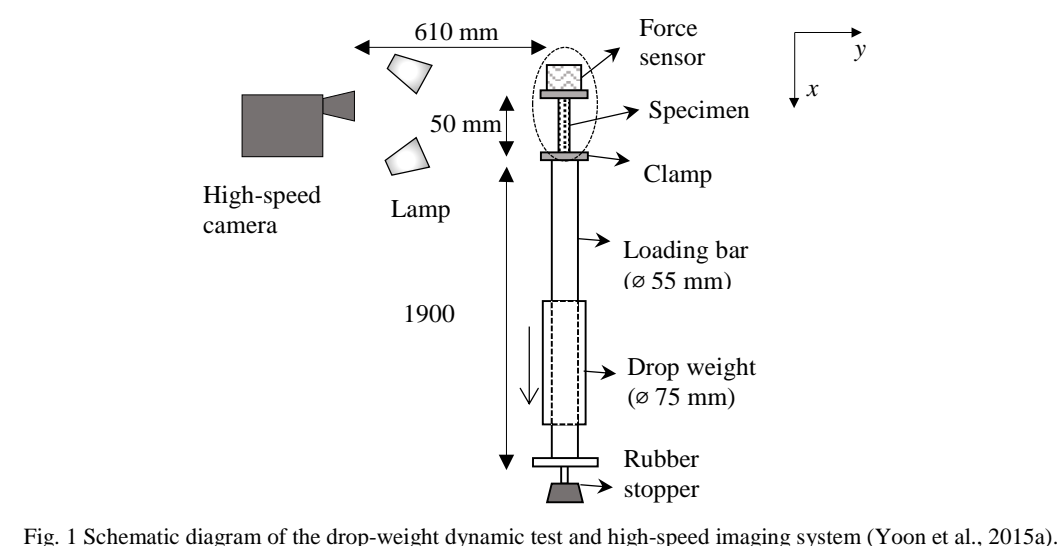

load is applied at both ends of a polyurea specimen. All of these studies assumed that the nonequilibrium state is limited to a very early portion of the loading. However, with the advent of low noise, high resolution high speed imaging, an alternative approach may be adopted, namely, to directly use the non-equilibrium state caused by wave propagation in a specimen in order to obtain a dynamic stress-strain curve. This has been done for shock waves using the nonlinear onedimensional wave equation and jump conditions (Niemczura and Ravi-Chandar, 2011) and, more recently, by the present authors for lower amplitude stress waves with full-field (two dimensional) surface measurements of the specimen response, the basis of the technique used in the current paper (Yoon et al., 2015a).

In order to extract material response from measurements of stress wave propagation in materials, a suitable data analysis procedure is required. The present authors performed this analysis using the Virtual Fields Method (VFM) (Pierron and Grédiac, 2012). The VFM is an inverse technique that makes use of the Principle of Virtual Work equation (PVW). The use of this equation with a set of experimental data such as strain and force allows inverse identification of parameters of an assumed constitutive equation (the method may also be used to both identify and asses the applicability of different constitutive models). For high speed experiments, the dynamic VFM may be used, in which the PVW is manipulated so that the traction force term (i.e. virtual work due to external forces) is cancelled. This cancellation means that force data are no longer required; instead, the virtual work done by inertial forces (i.e. acceleration data) is used as a virtual load cell. A description of this technique is given in detail in a recent paper (Pierron et al., 2014) introducing the application of the dynamic VFM to data obtained from a fibre-reinforced composite. The present authors have used the dynamic VFM to identify Young's modulus of rubbers under medium strain rate loading ( $100 \mathrm{~s}^{-1}$ in tension) produced by a drop-weight apparatus schematically described in Fig. 1. The dynamic full-field data (strain and acceleration) were obtained by means of a high-speed camera (FASTCAM SA5, Photron) and the digital image correlation (DIC) technique. In this apparatus, the strain amplitudes of the dynamic loading were too small to obtain both the modulus and strain hardening parameter for the assumed one-term Ogden constitutive model. Instead, a series of experiments were performed in which the specimen was statically pre-loaded to a number of different strains before the dynamic loading took place. The data were then analysed using a rateindependent isotropic linear elastic model applied in the PVW. Hence, a series of dynamic moduli was obtained; these were assumed to be secant moduli of the material's stress-strain curve. The moduli were then used in an optimization procedure in order to identify parameters of the 
hyperelastic material. The same drop-weight and imaging procedures were identically adopted for the present work, although the analysis is very different.

The dynamic VFM is based on the utilization of acceleration fields, which are generated when specimens are subjected to a non-uniform deformation. This means that quantities including strain, displacement and acceleration, spatially vary during an experiment. This variation can also include strain rate fields, although in the previous paper (Yoon et al., 2015a) the strain rate for a particular drop-weight test was obtained by spatial averaging. The existence of the variation of strain rate has the potential to give further information about the material response, in particular the rate dependence. Moreover, in the previous drop-weight experiments, only the initial loading period was adopted for the analysis of the VFM; in fact, multiple reloading takes place in a single drop-weight experiment owing to wave reflection at the specimen clamp interfaces, which overall explores a larger deformation range. There are further reloading events due to wave reflection in the loading bar. The multiple reloading data can allow consideration of the stress-strain nonlinearity and, simultaneously, the rate-dependent behaviour, i.e. viscoelastic characteristics. Using these two observations, (1) strain rate variation during a particular loading step and (2) multiple reloading data, this paper presents the calibration of a viscoelastic model by applying the VFM to drop-weight experiments on two rubbers. In another recent paper, the present authors demonstrated a method to apply a nonlinear constitutive model in the dynamic VFM to obtain large amplitude data suitable for the characterisation of a rate independent hyperelastic model (Yoon et al., 2015b). The framework of this nonlinear (dynamic) VFM is adopted in the present paper and will be described later. Few previous studies have explored the application of the VFM for the viscoelastic behaviour of soft materials. Sasso et al. (2013) utilized the nonlinear VFM in order to identify inelastic behaviour of a rubber, but the parameters of the viscoelastic model (Prony series, which is defined as Eq. (2) in the simulation section of the current paper) used were calibrated separately from the VFM calculations. Connesson et al. (2015) have shown that the dynamic VFM was able to identify viscoelastic parameters based on a frequency scale for a soft biological material. In the present paper, the application of the nonlinear VFM to the viscoelastic behaviour is used to identify the parameters of a Prony series model and reconstruct a stress relaxation curve from data obtained under dynamic loading.

The range of frequencies over which viscoelastic behaviour can be observed during the dropweight experiment is limited by the time scale of the total data and also the imaging speed. Thus, these two factors limit the time range of a reconstructed stress relaxation curve. In order to extend this limitation, the present paper also explores the application of the time temperature superposition principle (TTSP) to the drop-weight experiment. A temperature controlling device is introduced to the drop-weight apparatus used previously (Yoon et al., 2015a) so that each relaxation curve, constructed by the Prony parameters obtained at different temperatures, can be shifted using the principle of time-temperature superposition.

A well-established technique to obtain temperature and frequency dependence of polymer moduli is Dynamic Mechanical Analysis (DMA). This paper starts with the description of the DMA data and relaxation curves of the nitrile and silicone rubbers used in this study. A Prony series model is fitted to these two relaxation curves to obtain their parameters. The obtained parameters are applied to FEM simulations of the drop-weight experiment. Simulation data are analysed by the nonlinear VFM to identify the relaxation curve at a particular temperature. Each relaxation curve is shifted to build a master curve, which is then compared to the given relaxation behaviour. Experiments are then performed on the two materials, and the same analysis procedure is applied; here, the reconstructed relaxation curves are compared to those obtained from the DMA technique. Hence, for these two model materials, the new dynamic technique will be validated against a wellestablished method, giving confidence for the application to a wider range of different polymers and polymer based systems.

Commented [SY2]: Reviewer 2

Page 3. They mention the 'Prony series' concept several page before they define what it is (which they do on page 7). Perhaps they could either give a reference on page 3 or state that it will be defined later in the paper. 


\section{DMA tests on nitrile and silicone rubbers}

Two model materials were used in this study: nitrile (Coru118, Coruba) and silicone rubbers (Sylgard 184 silicone elastomer, Dow corning). The nitrile rubber was supplied as a thin sheet; the silicone rubber was cured as described in Yoon et al. (2015a). Although DMA experiments on the silicone rubber have already been reported, an improved control mode was adopted in the present study, so the material was re-tested. DMA experiments were performed in a TA Instruments Q800
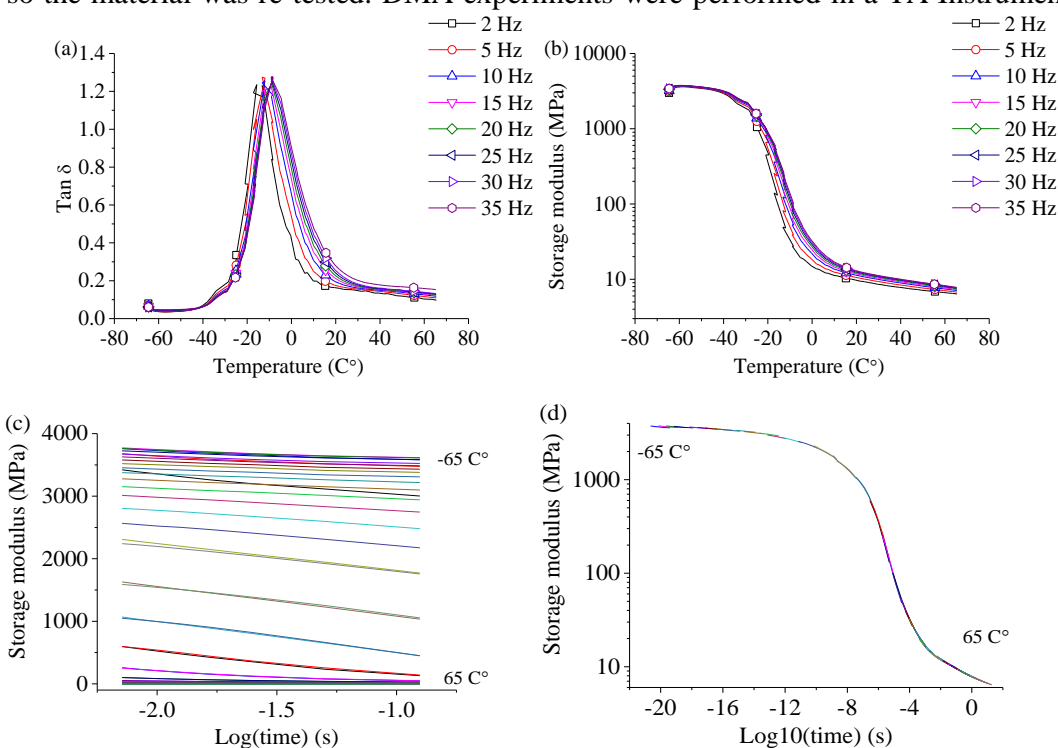

Fig. 2 Nitrile rubber: (a) storage moduli and (b) $\tan \delta$ versus temperature at the five frequencies; (c) isotherm of storage modulus and (d) master curve versus the logarithmic time. 

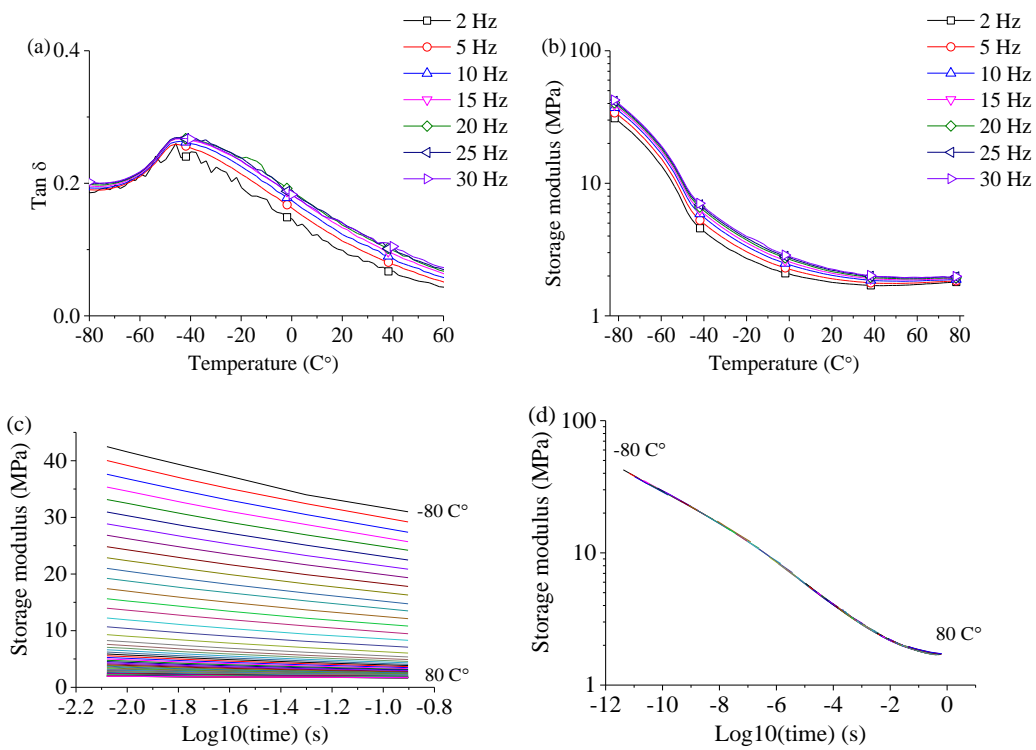

Fig. 3 Silicone rubber: (a) storage moduli and (b) $\tan \delta$ versus temperature at the four frequencies; (c) isotherm of storage modulus and (d) master curve versus the logarithmic time.

DMA system, using temperature steps and frequency sweeps in a film tension mode. For the nitrile rubber, isothermal frequency sweeps were performed at different temperatures $\left(-65\right.$ to $65{ }^{\circ} \mathrm{C}$ in $2{ }^{\circ} \mathrm{C}$ increments); the isothermal duration was given as $1 \mathrm{~min}$ for each step; and five frequencies were applied: 2, 5, 10, 15, 20, 30 and $35 \mathrm{~Hz}$. The silicone rubber was tested similarly, but with a temperature range -80 to $80{ }^{\circ} \mathrm{C}$ and applying only the first four frequencies. In order to obtain relaxation curves, the well-established time temperature superposition procedure was applied, as described in Yoon et al. (2015a), using the isotherm curves obtained at each temperature. The DMA results from the two rubbers are presented in Fig. 2 and Fig. 3. The relaxation curves of Fig. 2(d) and Fig. 3(d), produced as a result of the time-temperature superposition were used to provide material responses in the simulation and experimental work described in the later sections. 


\section{FINITE ELEMENT SIMULATION}

\subsection{Simulation configuration}
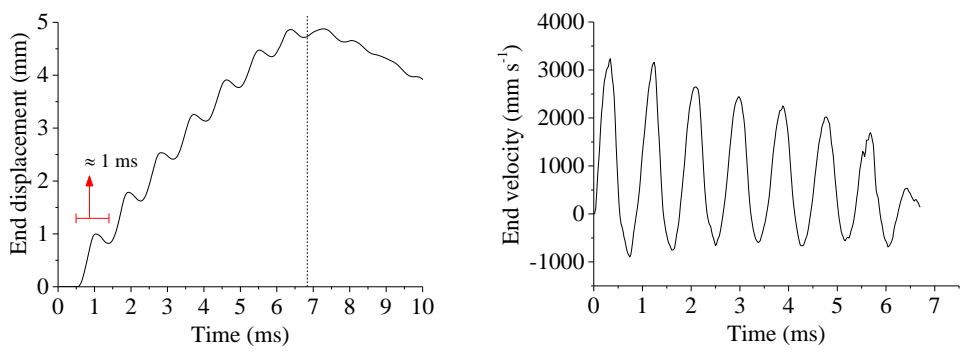

Fig. 4 (a) End displacement and (b) velocity profile of the drop-weight test on a nitrile rubber (the dash line indicates the final data range for the present simulation study).

Before conducting experimental work, finite element simulations were used to produce model output data to develop and test the VFM analysis. Firstly, it is necessary to define the time scale over which relaxation data can be obtained, which is done by consideration of experimental data. The experiment procedure including the application of DIC is explained in the previous paper (Yoon et al., 2015a). The lower limit of the time scale of the relaxation behaviour is limited by the imaging speed. A speed of 50000 frames per second (fps), which was adopted in the previous work (Yoon et al., 2015a), was also used in the present experiments; thus, the time step $\Delta t$ between each picture is $2 \times 10^{-5} \mathrm{~s}$, and in a logarithmic scale $-4.77\left(\approx \log _{10}\left(2 \times 10^{-5}\right)\right)$. It is therefore assumed that the relaxation behaviour associated with logarithmic time scales smaller than -4.7 is impossible to characterize because its physical behaviour is not observed. With regard to the upper limit, the form of the displacement provided by the lower clamp to the specimen is shown in Fig. 4(a). The reloading is caused by oscillation of the stress wave induced in the loading bar by the drop-weight. It is assumed that the relaxation behaviour that can be characterized by the dynamic VFM must be clearly exhibited within each reloading period because the non-uniform deformation state (consequently, acceleration field) is re-generated when each reloading is initiated. The period of each reloading is approximately $1 \mathrm{~ms}$, as indicated in Fig. 4(a) showing end displacement data obtained from an experiment on a nitrile rubber. The logarithmic time of $1 \mathrm{~ms}$ is -3 , which is used for the upper limit of the time scale.

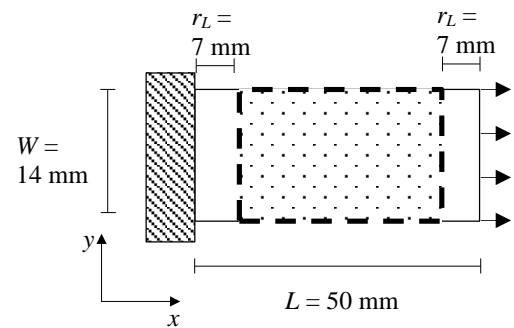

Fig. 5 Two-dimensional simulation geometry: the right (hatch); the dotted rectangle indicates the data area used for the nonlinear VFM.

The overall simulation procedure is the same as used previously (Yoon et al., 2015a). ABAQUS/Explicit simulations were conducted with a CPS4R (four-node plane stress) type element, an element size of $0.2 \mathrm{~mm}$ and a data interval $20 \mu$ s to resemble the imaging speed of $50000 \mathrm{fps}$. The simulation specimen dimensions were $50 \mathrm{~mm}$ long and $14 \mathrm{~mm}$ wide, which is similar to the actual specimen size. The simulation geometry is described in Fig. 5. The left- and right-hand sides are respectively subjected to fixed and velocity boundary conditions; the velocity 
profile shown in Fig. 4(b) is used. The right-hand side edge is also fixed in the transverse direction to mimic the effect of the clamps used in the actual experiment. The total simulation duration is approximately $7 \mathrm{~ms}$, at which global unloading starts to occur as indicated by a dash line in Fig. 4(a).

The relaxation behaviour can be described in ABAQUS by a Prony series model. Prior to applying this model, the master relaxation curves (shown in Fig. 2(d) and Fig. 3(d)) were smoothed by fitting their shift factor $a_{\mathrm{T}}$ to the WLF (Williams-Landel-Ferry) model (Williams et al., 1955), given as

$$
\log _{10} a_{\mathrm{T}}=-\frac{C_{1}\left(\mathrm{~T}-\mathrm{T}_{0}\right)}{C_{2}+\left(\mathrm{T}-\mathrm{T}_{0}\right)},
$$

where $\mathrm{T}$ is the temperature and $\mathrm{T}_{0}$ is a reference temperature $\left(20^{\circ} \mathrm{C}\right) ; C_{1}$ and $C_{2}$ are material constants. The shifting factors were manually determined so that each isotherm is well overlapped. Then, the manual shifting factors and the temperatures of each isotherm were fitted by Eq. (1) in order to obtain optimized $C_{1}$ and $C_{2}$ parameters. The manual shifting factors and the WLF model with the optimized parameters $\left(C_{1}\right.$ and $\left.C_{2}\right)$ are shown as a function of temperature in Fig. 6 . The values of $C_{1}$ and $C_{2}$ are given in the legends. The WLF model was then used to generate the master curves given in Fig. 7. These figures are plotted in a normalized form, and for the silicone rubber, the relaxation data below $\tau=1 \times 10^{-7} \mathrm{~s}$ are excluded as they are not fitted well by the WLF model. A similar procedure was introduced in a previous study (Kaliske and Rothert, 1997).
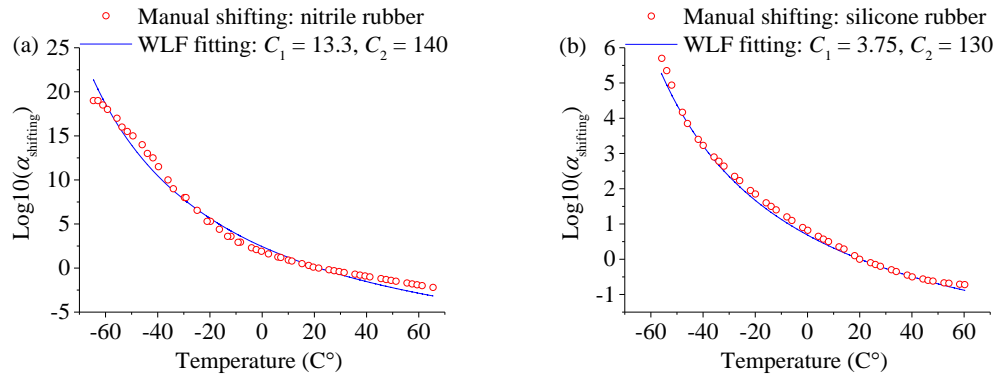

Fig. 6 WLF model fitting on the manual shifting factor versus temperature profiles of the nitrile (a) and silicone (b) rubbers.
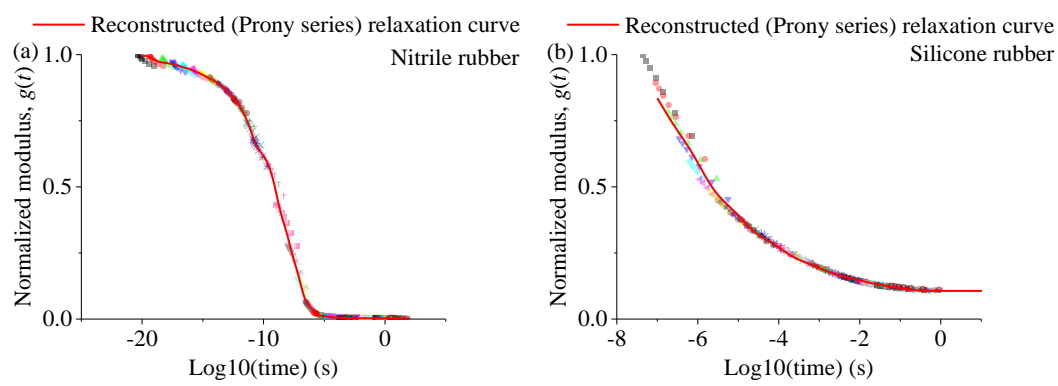

Fig. 7 Normalized relaxation curves of the DMA relaxation data (scattered data) and the fitted profiles using the Prony series (red solid line). 
Table 1 Prony series parameters: given relaxation time terms $\tau$ and optimized normalized-relaxation terms $g$ obtained from the DMA data of the nitrile and silicone rubbers.

\begin{tabular}{|c|c|c|c|c|c|c|c|c|c|c|c|}
\hline \multicolumn{12}{|c|}{ Nitrile rubber } \\
\hline $\log 10\left(\tau_{i}\right)$ & -19 & -18 & -17 & -16 & -15 & -14 & -13 & -12 & -11 & -10 & -9 \\
\hline$\overline{g_{i}}$ & 0.028 & 0.004 & 0.014 & 0.014 & 0.024 & 0.020 & 0.046 & 0.047 & 0.149 & 0.027 & 0.201 \\
\hline $\log 10\left(\tau_{i}\right)$ & -8 & -7 & -6 & -5 & -4 & -3 & -2 & -1 & 0 & & \\
\hline$g_{i}$ & 0.156 & 0.198 & 0.056 & 0.010 & 0.003 & 0.001 & 0.001 & 0.0002 & 0.001 & & \\
\hline \multicolumn{12}{|c|}{ Silicone rubber } \\
\hline $\log 10\left(\tau_{i}\right)$ & -7 & -6 & -6 & -4 & -3 & -2 & -1 & & & & \\
\hline$g_{i}$ & 0.216 & 0.280 & 0.163 & 0.103 & 0.066 & 0.038 & 0.029 & & & & \\
\hline
\end{tabular}

The Prony series parameters were then obtained by fitting these relaxation curves. A Prony series model describes a relaxation modulus $E(t)$ by the following form

$$
E(t)=E_{0}-\sum_{j=1}^{m} E_{j}\left(1-\exp \left(-t / \tau_{j}\right)\right),
$$

where $E_{0}, t$ and $m$ denote the instantaneous modulus, time and the total number of terms; $E_{j}$ and $\tau_{j}$ are the relaxation modulus and relaxation time for each Prony series term. The normalized form of this function is frequently used for a numerical formulation:

$$
\begin{gathered}
E(t) / E_{0}=1-\sum_{j=1}^{m} E_{j} / E_{0}\left(1-\exp \left(-t / \tau_{j}\right)\right), \\
g(t)=1-\sum_{j=1}^{m} g_{j}\left(1-\exp \left(-t / \tau_{j}\right)\right) .
\end{gathered}
$$

The normalized relaxation curves in Fig. 7 were fitted by Eq. (4) with fixed relaxation times $\left(\tau_{1}=1 \times 10^{-19}, \tau_{2}=1 \times 10^{-18}, \ldots, \tau_{20}=1 \mathrm{~s}\right)$ for the nitrile rubber and $\left(\tau_{1}=1 \times 10^{-7}, \tau_{2}=1 \times 10^{-6}, \ldots\right.$, $\left.\tau_{7}=1 \mathrm{~s}\right)$ for the silicone rubber in order to obtain the corresponding normalized modulus terms, $g_{j}$. The fitting was conducted using the MATLAB built-in function, fmincon, with a lower bound of $1 \times 10^{-10}$ to ensure positive values of $g_{j}$. The two sets of normalized modulus terms are listed in 
Table 1, and these were used to calculate the reconstructed curves presented in Fig. 7 as a solid line. These two sets of viscoelastic properties were used in the subsequent simulation work.

The relaxation behaviours for the two simulation cases are now defined. Their long-term behaviours, i.e. when the materials are full relaxed $(t=\infty)$, were assumed to be described by the one-term Ogden model (Ogden, 1972), the strain energy density of which in ABAQUS (ABAQUS, 2011) is written as

$$
W=\frac{2 \mu}{\alpha}\left(\lambda_{1}^{\alpha}+\lambda_{2}^{\alpha}+\lambda_{3}^{\alpha}-3\right)
$$

where $\mu$ and $\alpha$ are material parameters, and $\lambda_{i}(i=1,2,3)$ are principal stretch ratios. It should be noted that only a small deformation range, which can be produced by the drop-weight experiment, is explored in the present study. Therefore, the use of the one-term Ogden model is sufficient to describe the behaviour of the rubbers, where the nonlinearity of a stress-strain relationship is less complex. In ABAQUS, it is assumed that $\mu$ is the only rate-dependent term, and $\alpha$ is constant. The long $(t=\infty)$ and instantaneous $(t \approx 0) \mu$ terms have the following relationship

$$
\mu_{\infty}=\mu_{0}\left(1-\sum_{j=1}^{m} g_{j}\right) .
$$

For the $\mu_{\infty}$ term, a quasi-static uniaxial test was conducted at a low strain rate of $10^{-5} \mathrm{~s}^{-1}$ on each rubber. The experimental procedure described in (Yoon et al., 2015b) was adopted. The total strain was limited to 0.001 true strain up to which the stress-strain behaviour was observed to be almost linear. The experimental results are shown in Fig. 8, in which the linear fits are also provided. The slopes of the linear fits to the silicone and nitrile rubbers are obtained as 1.11 and $2.83 \mathrm{MPa}$ respectively. These values are taken as estimates of the long-term Young's moduli. The $\mu_{\infty}$ terms can be taken as the initial long-term shear modulus; thus, they were obtained by dividing the Young's moduli by 3 , using the assumption of incompressibility. The values of $\mu_{\infty}$ are 0.37 and $0.94 \mathrm{MPa}$ respectively for the silicone and nitrile rubbers. The $\alpha$ term is assumed to be 4 for both cases. The values of density were given as $1480 \mathrm{~kg} \mathrm{~m}^{-3}$ (nitrile rubber) and $1200 \mathrm{~kg} \mathrm{~m}^{-3}$ (silicone rubber).

It is assumed that the temperature dependence of the simulation materials is described by the WLF model. The parameters of $C_{1}$ and $C_{2}$ obtained through the optimization procedure explained above were given to the simulations. Other thermal properties are ignored as the heat transfer is not important in the current simulation. Also, the stress variation due to thermal expansion is not considered because, in the actual experiment, the specimen is clamped only after being allowed to equilibrate at the required temperature level. In total 19 temperatures were applied over the whole simulation geometry as an initial condition, ranging from -12 to $60^{\circ} \mathrm{C}$ in intervals of $4{ }^{\circ} \mathrm{C}$. In the actual experiment, only part of a specimen surface was covered by the temperature controlling device, which will be mentioned in the experiment section. This experiment condition was also considered in the simulation by reducing the data analysis area by $7 \mathrm{~mm}$ from both ends, as described in Fig. 5. 

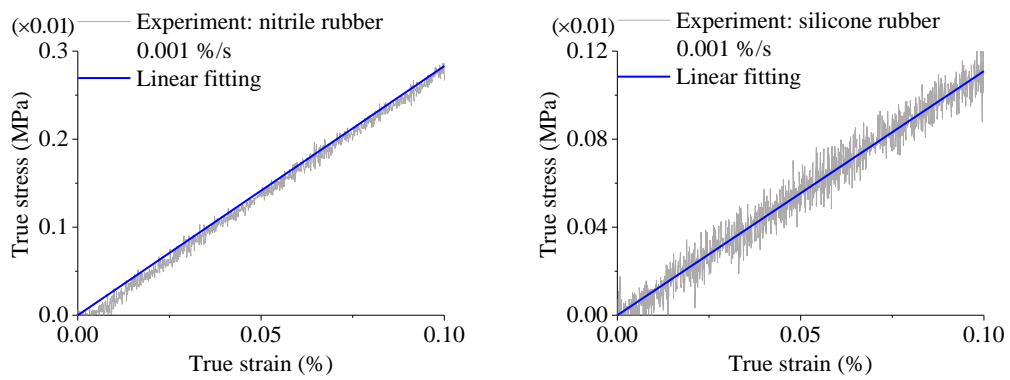

Fig. 8 Quasi-static uniaxial tests at a strain rate of $0.001 \% / \mathrm{s}$ on the nitrile and silicone rubbers.

3.2. VFM analysis

The nonlinear VFM introduced in a previous paper (Yoon et al., 2015b) is adopted. The PVW, based on the initial configuration and the first Piola-Kirchhoff stress $\Pi$, can be written as (Pierron and Grédiac, 2012)

$$
-\int_{\mathrm{V}_{0}} \boldsymbol{\Pi}: \frac{\partial \boldsymbol{u}_{0}^{*}}{\partial \mathbf{x}_{0}} \mathrm{dV}_{0}+\int_{S_{0, f}}(\boldsymbol{\Pi} \boldsymbol{N}) \cdot \boldsymbol{u}_{0}^{*} \mathrm{~d} S_{0}=\int_{\mathrm{V}_{0}} \rho \boldsymbol{a} \cdot \boldsymbol{u}_{0}^{*} \mathrm{dV}_{0} .
$$

$\begin{array}{ll}\boldsymbol{\Pi} & \text { the first Piola-Kirchhoff stress tensor } \\ \mathrm{V}_{0} & \text { initial volume of the body } \\ S_{0, f} & \text { initial loaded surface } \\ \mathbf{x}_{0} & \text { initial coordinate } \\ \boldsymbol{N} & \text { initial normal vector of } S_{0, f} \\ \boldsymbol{u}_{0}^{*} & \text { virtual displacement vector based on the initial coordinate } \\ \boldsymbol{\varepsilon}_{0}^{*} & \text { virtual strain tensor }\left(=\partial \boldsymbol{u}_{0}^{*} / \partial \mathbf{x}_{0}\right) \\ \rho & \text { density } \\ \boldsymbol{a} & \text { acceleration vector }\end{array}$

A similar form of this PVW has been used in several previous VFM studies on quasi-static deformation of rubbers (Promma et al., 2009; Sasso et al., 2013). This PVW can be rewritten to remove the traction force term by means of the following virtual field (Moulart et al., 2011)

$$
\left\{\begin{array} { l } 
{ u _ { x , 0 } ^ { * } = x ( x - L ) } \\
{ u _ { y , 0 } ^ { * } = 0 }
\end{array} \Rightarrow \left\{\begin{array}{l}
\frac{\partial u_{x, 0}^{*}}{\partial x_{0}}=2 x-L \\
\frac{\partial u_{y, 0}^{*}}{\partial y_{0}}=0 \\
\frac{\partial u_{x, 0}^{*}}{\partial y_{0}}=0, \frac{\partial u_{y, 0}^{*}}{\partial x_{0}}=0
\end{array},\right.\right.
$$

whilst the assumption of plane stress leads to the following PVW

$$
-\int_{S_{0}} \Pi_{x x} \frac{\partial u_{x, 0}^{*}}{\partial x_{0}} \mathrm{~d} S_{0}=\int_{S_{0}} \rho a_{x} u_{x, 0}^{*} \mathrm{~d} S_{0} .
$$

The stress term $\Pi_{x x}$ is described by the one-term Ogden model for a hyperelastic material combined with the Prony series model for the viscoelasticity. The combination of these two models was given by the formulation initially proposed by Simo (1987); this method was similarly adopted in a previous VFM study (Sasso et al., 2013). In the present work, it is assumed that the global experiment coordinates and principal directions are coincident $\left(\lambda_{x}=\lambda_{1}\right)$. First, the instantaneousand long-term hyperelastic behaviours are given as, in terms of true stress, 


$$
\begin{gathered}
\sigma_{x, 0}=\frac{2 \mu_{0}}{\alpha}\left(\lambda_{x}^{\alpha}-\lambda_{x}^{-0.5 \alpha}\right), \\
\sigma_{x, \infty}=\frac{2 \mu_{\infty}}{\alpha}\left(\lambda_{x}^{\alpha}-\lambda_{x}^{-0.5 \alpha}\right),
\end{gathered}
$$

where the subscripts ' 0 ' and ' $\infty$ ' respectively indicate the instantaneous and long-term values. For the $\alpha$ term, there is no indication by any subscript as it is assumed that the same $\alpha$ is shared between these two behaviours. The total stress at a time step $n$ is expressed using the following formulation

$$
\begin{gathered}
\sigma_{x, n}^{i}=\sigma_{x, n-1}^{i} \exp \left(-\frac{\Delta t}{\tau_{i}}\right)+g_{i} \exp \left(-\frac{\Delta t}{2 \tau_{i}}\right)\left(\sigma_{x, 0, n+1}-\sigma_{x, 0, n}\right), \\
\sigma_{x, n}=\sigma_{x, \infty, n}+\sum_{i} \sigma_{x, n}^{i},
\end{gathered}
$$

where ' $i$ ' is the number of the terms involved in the Prony series model. In total, five terms were used, and, for each term, the following relaxation time terms were assumed using the definition of the upper and lower time scale range: $\tau_{1,2,3,4,5}=1 \times 10^{-5}, 5 \times 10^{-5}, 1 \times 10^{-4}, 5 \times 10^{-4}$ and $1 \times 10^{-3} \mathrm{~s}$. The total stress $\sigma_{x x}$ is converted to $\Pi_{x x}$ using the relation $\Pi_{x x}=\sigma_{x x} / \lambda_{x}$, which is then applied in the PVW, Eq. (9). The acceleration term $\boldsymbol{a}$ used in this equation is obtained by applying the following finite different equation on displacement fields

$$
\frac{\boldsymbol{u}(t+\Delta t)+\boldsymbol{u}(t-\Delta t)-2 \boldsymbol{u}(t)}{\Delta t^{2}}=\boldsymbol{a}(t),
$$

the density $\rho$ is assumed to be known; and the strain $\varepsilon$ is numerically calculated by applying a usual strain-displacement matrix on $\boldsymbol{u}$.

The PVW is calculated with a set of numerical data, $\mathbf{x}(t)$ and $\boldsymbol{u}(t)$, to identify material parameters involved in the constitutive equation. As mentioned, the linear and nonlinear VFMs are defined when the stress term in their PVW is given respectively by linear and nonlinear constitutive equations. For example, the linear VFM can be set up when the linear elastic constitutive equation is used. The calculation of the PVW can be conducted by making a system of linear equations and linearly solving it to find Young's modulus and Poisson's ratio. In contrast, it is difficult to linearly solve the nonlinear VFM as material parameters are nonlinearly dependent on deformations. The present VFM in which the PVW is combined with Eq. (13) was nonlinearly calculated by setting up and minimizing the following cost function

$$
\Phi=\sum_{k=1}^{n}\left[\int_{S_{0}} \Pi_{x x}\left(t_{k}\right) \frac{\partial u_{x, 0}^{*}}{\partial x_{0}} \mathrm{~d} S_{0}+\int_{S_{0}} \rho a_{x}\left(t_{k}\right) u_{x, 0}^{*} \mathrm{~d} S_{0}\right]^{2},
$$

where $n$ is the total number of time steps. The minimization of this cost function was conducted in the same way as used for the identification of the Prony series model. After this calculation, the parameters to be determined are $g_{i}$, corresponding to each $\tau_{i}, \alpha$ and $\mu_{0}$; the $\mu_{\infty}$ term is determined by the relation of $\mu_{\infty}=\mu_{0} \times\left(1-\Sigma g_{i}\right)$. The identified $\mu_{0}$ and $\mu_{\infty}$ do not represent the instantaneous and long-term shear modulus of the materials in an actual time scale but in the defined drop-weight experiment time scale. For comparison, the linear elastic constitutive model was also used. The same formulation was used with the following constitutive relations

$$
\begin{gathered}
\sigma_{x, 0}=Q_{x x, 0} \varepsilon_{x x}+Q_{x y, 0} \varepsilon_{x y}, \\
\sigma_{x, \infty}=Q_{x x, \infty} \varepsilon_{x x}+Q_{x y, \infty} \varepsilon_{x y}, \\
Q_{x x, \infty}=E_{\infty} /\left(1-v^{2}\right), Q_{x y, \infty}=v E_{\infty} /\left(1-v^{2}\right) \\
Q_{x x, 0}=E_{\infty} /\left(1-v^{2}\right), Q_{x y, 0}=v E_{0} /\left(1-v^{2}\right)
\end{gathered}
$$

These equations were applied to Eq. (12) and (13) for the viscoelastic behaviour. The Poisson's ratio, $v$, in this equation is assumed to be 0.5 . 
3.3. Simulation results
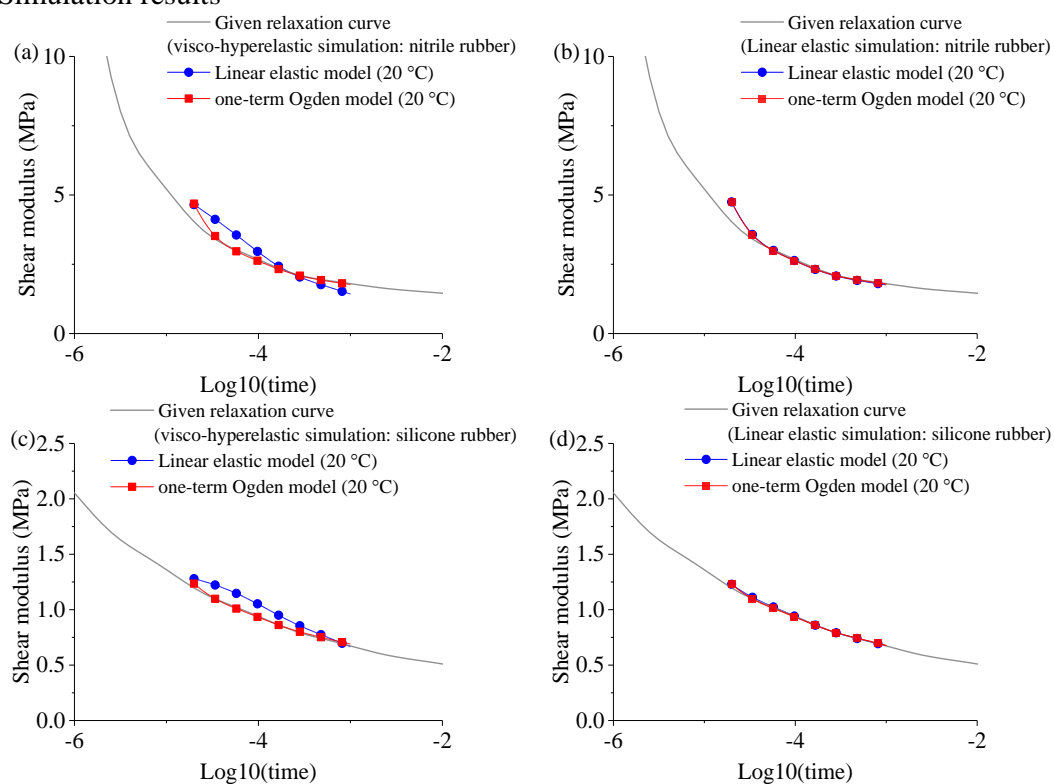

Fig. 9 Identification of a local relaxation curve from the nonlinear VFM using the linear elastic and one-term Ogden model on the simulation data with the nitrile and silicone rubber relaxation behaviour. Using the noise study method described in (Yoon et al., wacement data polluted by white Gaussian noise with the actual experimental noise amplitude, $0.5 \times 10^{-3} \mathrm{~mm}$, were also used in the same VFM analysis. However, the deviation from the result shown above is small enough so that error bars are located within the symbols. Thus, error bars are omitted in this figure.

The nonlinear VFM described above was applied to the simulation data given by the one-term Ogden model with the two relaxation behaviours representing the two materials. The identified parameters for these two cases are listed in Table 2. Using these identified parameters, the local relaxation curves were reconstructed. First, the normalized local relaxation curve was plotted using Eq. (4) and multiplied by the instantaneous shear modulus term $\mu_{0}$. The reconstructed relaxation curves from the two simulation cases are presented in Fig. 9(a and c) and indicated by a redrectangle symbol. The same reconstruction procedure was used on the parameters identified from the nonlinear VFM with the linear elastic model. One difference is that value of $\mu_{0}$ to be multiplied to the normalized curve is obtained by dividing the identified $E_{0}$ by 3 . Its results are presented by a curve with blue-circular symbols. Fig. 9(a and c) clearly shows the difference between the two nonlinear VFMs in that the use of the one-term Ogden model gives a better matching to the part of the given relaxation curves. The results in Fig. 9(b and d) were obtained by the same nonlinear VFM on the simulation conducted with the linear elastic model instead of the Ogden model by converting the given $\mu_{\infty}$ to $E_{\infty}$ and $v=0.499$. In this simulation case, the two reconstructed curves are almost matched. The comparison between Fig. 9(a and c) and Fig. 9(b and d) indicates that in the present nonlinear VFM application for the viscoelastic behaviour of rubbers, the use of a nonlinear constitutive model can give a better identification result.

Table 2 Identified Ogden and Prony model parameters from the viscoelastic simulations at $20^{\circ} \mathrm{C}$

\begin{tabular}{lc|cc}
\hline \multicolumn{2}{c|}{$\begin{array}{c}\text { Nitrile rubber } \\
\text { Simulation at } 20^{\circ} \mathrm{C}\end{array}$} & \multicolumn{2}{|c}{$\begin{array}{c}\text { Silicone rubber } \\
\text { Simulation at } 20^{\circ} \mathrm{C}\end{array}$} \\
\hline$\mu_{0}(\mathrm{MPa})$ & 13.3 & $\mu_{0}(\mathrm{MPa})$ & 2.10 \\
\hline
\end{tabular}




\begin{tabular}{cc|cc}
\hline$\alpha$ & 3.42 & $\alpha$ & 3.55 \\
\hline$g_{1}$ & 0.719 & $g_{1}$ & 0.431 \\
\hline$g_{2}$ & 0.027 & $g_{2}$ & 0.042 \\
\hline$g_{3}$ & 0.087 & $g_{3}$ & 0.112 \\
\hline$g_{4}$ & 0.024 & $g_{4}$ & 0.041 \\
\hline$g_{5}$ & 0.022 & $g_{5}$ & 0.072 \\
\hline
\end{tabular}

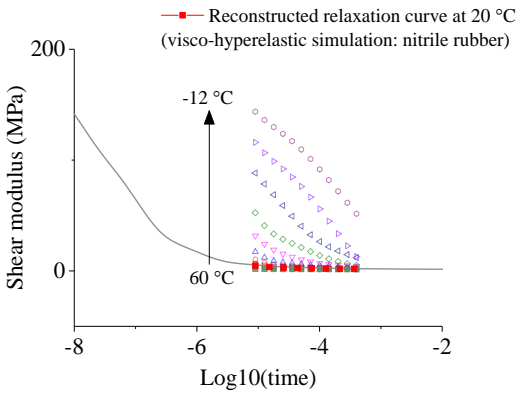

Fig. 10 Collection of the local reconstructed relaxation curves obtained from the nonlinear VFM applied on the viscoelastic simulations with the temperature range of -12 to $60^{\circ} \mathrm{C}$ with an interval of $4^{\circ} \mathrm{C}$.

The analysis results were obtained from the simulation data at $20^{\circ} \mathrm{C}$. The time span of the relaxation curve is limited by the time scales in the experiment as explained above. At this point, the time-temperature superposition principle (TTSP) was utilized in order to extend the length of the identified relaxation curves. The first step of the TTSP application is to collect the reconstructed local relaxation curves obtained at different temperatures. When TTSP was applied to the isotherm data obtained from the DMA test, manual shifting was conducted; the temperature step can be small enough so that there is significant overlap between data from adjacent temperature values, and this resemblance allows visual evaluation of the quality of the manual shifting. However, it was difficult to precisely control the temperature for the drop-weight experiment. In some cases, the local relaxation curve obtained may not have a good resemblance to the next curve or each curve could not be connected even after manual shifting because of the large temperature step. In order to overcome this expected experimental difficulty, the following automatic shifting procedure was adopted.

(1) The local relaxation curve at $20^{\circ} \mathrm{C}$ (red-rectangle symbol in Fig. 10) is fixed at its initial location. This reference curve is mathematically expressed as

$$
\begin{aligned}
& \hat{\mu}_{\left[20^{\circ} \mathrm{C}\right]}=f_{\text {Relaxation }}\left(\mu_{0,\left[20^{\circ} \mathrm{C}\right]}, g_{1, \ldots,\left[20^{\circ} \mathrm{C}\right]}, \widehat{t}_{[\text {fixed }]}\right) \\
& -4.7 \\
& \vdots \\
& \log 10\left(\widehat{t}_{\text {[fixed }]}\right)=\left\{\begin{array}{c}
\text { interval of } 0.01 \\
\vdots \\
-3
\end{array}\right\} .
\end{aligned}
$$

(2) The curve shifting is horizontally conducted; the shifting factor in this case is the logarithmic time, $\log 10\left(t_{\text {factor }}\right)$, denoted as $\beta_{\text {factor, }}$, and initialized to be zero. For example, when the relaxation curve at $16{ }^{\circ} \mathrm{C}$, which is the next step after $20^{\circ} \mathrm{C}$, is to be shifted, it can be expressed as

$$
\begin{aligned}
& \widehat{\mu}_{\left[16^{\circ} \mathrm{C}\right]}=f_{\text {Relaxation }}\left(\mu_{0,\left[16^{\circ} \mathrm{C}\right]}, g_{1 . \ldots,\left[16^{\circ} \mathrm{C}\right]}, \widehat{t}_{[\text {fixed }]}\right) . \\
& \log 10\left(\widehat{t}_{\left[16^{\circ} \mathrm{C}\right]}\right)=\log 10\left(\widehat{t}_{[\text {fixed }]}\right)+\beta_{\text {factor },\left[16^{\circ} \mathrm{C}\right]}
\end{aligned}
$$

(3) The data in Eq. (19)and (20) are collected in a vector form, written as

Commented [SY3]: Reviewer 2

"Page 11. They should insert '(TTSP)' after 'time-temperature superposition principle'" 


$$
\begin{aligned}
& \hat{\mu}=\left\{\begin{array}{l}
\hat{\mu}_{\left[16^{\circ} \mathrm{C}\right]} \\
\hat{\mu}_{\left[20^{\circ} \mathrm{C}\right]}
\end{array}\right\} \\
& \log 10(\hat{t})=\left\{\begin{array}{c}
\log 10\left(\widehat{t}_{\text {[fixed] }}\right)+\beta_{\text {factor }\left[16^{\circ} \mathrm{C}\right]} \\
\log 10\left(\widehat{t}_{\text {[fixed] }}\right)
\end{array}\right\}
\end{aligned}
$$

(4) It is assumed that the appropriate shifting of $\mu\left[16^{\circ} \mathrm{C}\right]$ with respect to $\mu\left[20^{\circ} \mathrm{C}\right]$ is achieved when $\beta_{\text {factor, }\left[16^{\circ} \mathrm{C}\right]}$ is found by which the data in Eq. (21) is well fitted to the one-term exponential model shown below

$$
\widehat{\mu}=a \exp (b \log 10(\widehat{t})) .
$$

The goodness of fit was evaluated by the value of the root-mean-square error, RMSE. This fitting procedure can be conducted by the built-in MATLAB function, fit, and RMSE can be also obtained from this function. This procedure to find a proper $\beta_{\text {factor, }\left[16^{\circ} \mathrm{C}\right]}$ can be expressed as

$$
\underset{\beta_{\text {factor }\left[16^{\circ} \mathrm{C}\right]}}{\min } \operatorname{RMSE}=f_{\text {exponential_fit }}\left(\widehat{\mu}, \log 10(\widehat{t}), \beta_{\text {factor }\left[16^{\circ} \mathrm{C}\right]}\right) .
$$

This minimization was conducted by using the fminunc function in MATALB. Once the curve of $\mu\left[16^{\circ} \mathrm{C}\right]$ is properly shifted, and this shifted data is used as a reference curve of the shifting of the next relaxation curve using the same procedure. A similar shifting procedure was used in the previous work of Shin and Tsai (1999).

Using the procedure explained above, the local curves at each temperature were shifted to extend the length of the relaxation curves. The results from the nitrile and silicone rubber simulations are presented in Fig. 11. The red-rectangle scatter line is the local relaxation curve obtained at $20{ }^{\circ} \mathrm{C}$. Starting from this reference curve, the shifting procedure was separately processed for the cold (20 to $-12{ }^{\circ} \mathrm{C}$ ) and hot temperature $\left(20\right.$ to $60^{\circ} \mathrm{C}$ ) ranges. The most right- and left-hand side curves are respectively obtained from the simulation data at 60 and $-12{ }^{\circ} \mathrm{C}$. As can be observed in this figure, the shifted data and the given nitrile and silicone relaxation curves are well matched. The present nonlinear VFM on the drop-weight data is able to identify the relaxation behaviour of rubber-like materials.
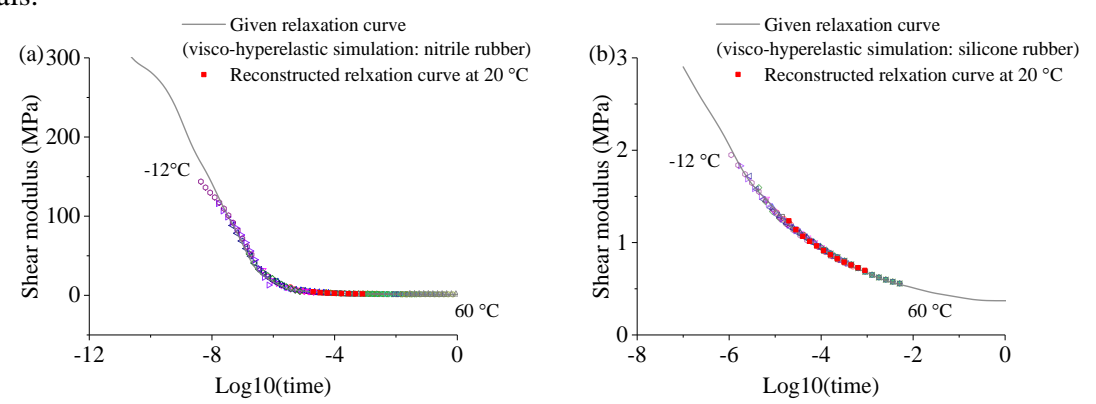

Fig. 11 Shifted local relaxation curves obtained from the drop-weight simulations at the temperature ranges from 60 to $-12{ }^{\circ} \mathrm{C}$ and the given nitrile and silicone rubber relaxation curves.

\section{EXPERIMENTAL IMPLEMENTATION}

4.1. Experimental procedure

The nitrile and silicone rubbers were tested using the drop-weight apparatus introduced in a previous paper (Yoon et al., 2015a). Temperature control was provided by a simple temperaturecontrolling device shown in Fig. 12, consisting of a cooling fan (TDEX3132, Thermo Electric Devices), Peltier stage (MCPF-127-14-11, Multicomp) and cuboidal copper block. A Peltier stage is a thermoelectric device which transfers heat from one side to the other when DC current is applied. The stage was attached to a heat sink so that the heat could be dissipated. The other side of the Peltier stage was bonded to the copper block by means of a thermal tape (Thermal Adhesive Tape 
8940, 3M). The other side of the copper block is also covered with the tape but with its coating film still in place in order to reduce friction between the specimen and the block. Temperature measurement was conducted using a K-type thermocouple, the data from which were collected by a USB thermocouple interface (USB TC01, National Instruments). Imaging illumination was provided by means of a continuous light lamp (Dedocool, DEDOTEC). The temperature increase during the duration of illumination (less than $20 \mathrm{~s}$ ) was measured to obtain an average temperature value, although this increase was observed to be less than $0.5^{\circ} \mathrm{C}$.

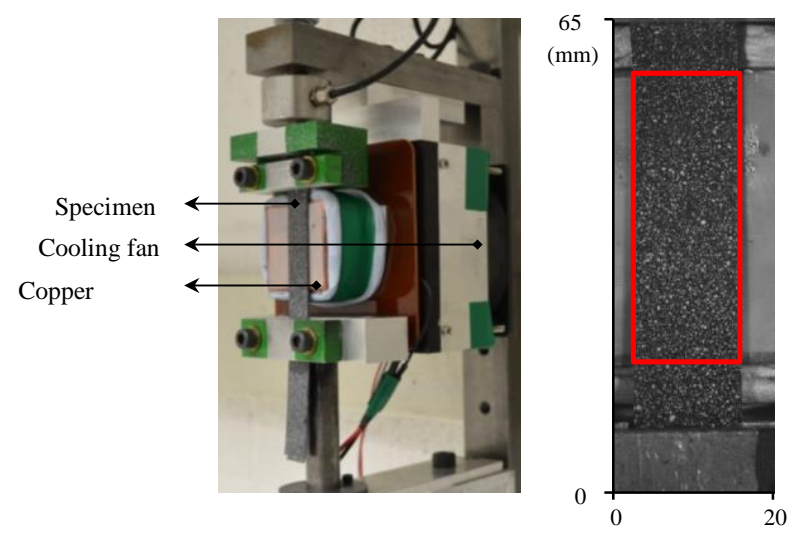

Fig. 12 (left) Temperature-controlling device installed on the drop-weight apparatus and (right) the in-situ high-speed image and the analysis area indicated by the red rectangle.

Table 3 Imaging and DIC analysis factors.

\begin{tabular}{c|c}
\hline camera & FASTCAM SA 5, Photron \\
\hline DIC software & Davis 7.2, LaVision 2007 \\
\hline field of view (data) & $533 \times 128$ pixels \\
\hline interframe time & $20 \mu \mathrm{s}$ \\
\hline subset size (final) & $12 \times 12$ \\
\hline subset overlap (final) & $75 \%$ \\
\hline spatial smoothing & not applied \\
\hline axial strain resolution & $1 \times 10^{-3}$ \\
\hline axial acceleration resolution & $7 \times 10^{5} \mathrm{~mm} \mathrm{~s}^{-2}$ \\
\hline
\end{tabular}

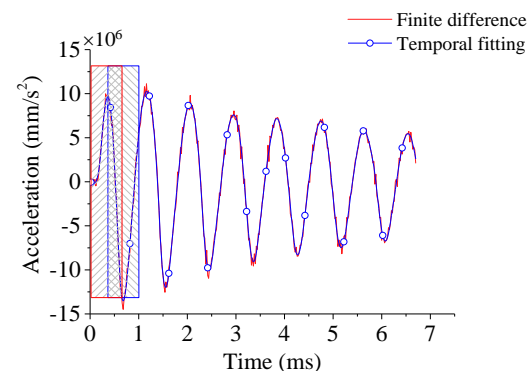

Fig. 13 Two spatially averaged acceleration profiles from the finite difference and temporal fitting calculation methods. 
The high-speed imaging and the DIC analysis were conducted as explained in the previous study. The imaging configuration is given in Table 3. As explained in the previous study, a temporal smoothing is able to alleviate the effect of noise on the parameter identification. Previously, the experimental acceleration data were obtained by applying temporal smoothing on the displacement data using a 9-degree polynomial. One difference in this present work is that the fitting was separately conducted on each reloading period. This procedure is visually described in Fig. 13, showing the two spatially averaged acceleration profiles. The red solid line is an acceleration profile obtained by the simple finite different method; the blue-circle line indicates the acceleration profile from the temporal fitting procedure. In this figure, the red- and blue-colour hatched rectangles represent the first and second period for the temporal fitting. The acceleration data obtained from these two separate periods were individually stored. It can be observed that half of the area of these two rectangles overlap each other. This overlapped area was divided into a half again; then, the acceleration data of the left-hand side of this overlapped area is given by the first period (red rectangle) and the second period (blue rectangle) data was used for the other side. Using this procedure, it was possible to remove spurious acceleration data that were calculated at the end of each averaging period. This separate averaging procedure was repeated for all loading periods.

4.2. Identification result

Table 4 provides the identified parameters for the nitrile and silicone rubbers and also the temperatures measured from each test. Using these parameters and the shifting procedure, the extended relaxation curves of the nitrile and silicone rubbers are given in Fig. 14. The relaxation data obtained from the DMA test are also presented in this figure; the DMA test was conducted in a tension mode so the storage moduli shown in Fig. 2(d) and Fig. 3(d) were divided by 3 to convert to the shear modulus. For both cases in Fig. 14, it can be seen that the shifted relaxation curves are well matched with the relevant DMA curves. However, further work is required to establish whether the differences between these curves are the result of fundamental physics of material behaviour, or are an artefact of the testing method. Also, the present nonlinear VFM can identify the different relaxation curve shapes (behaviour) of the nitrile and silicone rubbers. The identified values of $\mu_{0}$ in Table 4 explain this identification result in that the higher $\mu_{0}$ is identified for the lower temperature. The acceleration calculation methods are indicated in the legend of each figure; it seems that the effect of the acceleration fitting is not significant. 

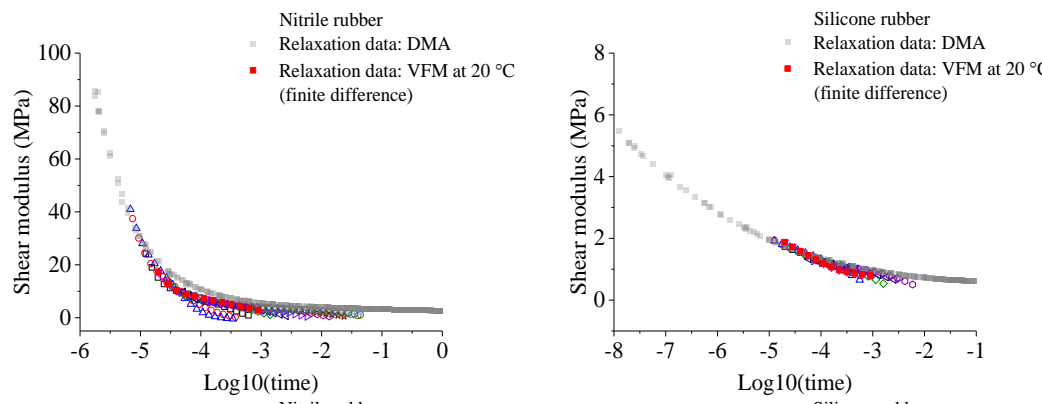

Nitrile rubber
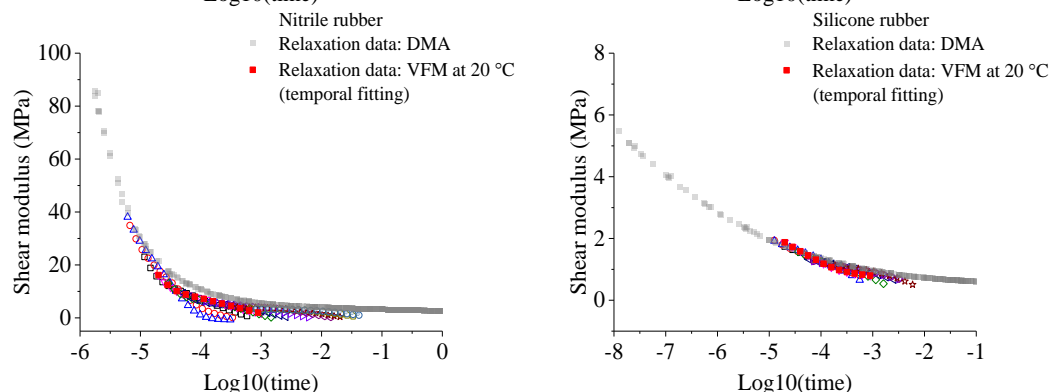

Fig. 14 Shifted local relaxation curves obtained from the drop-weight experiments and the nitrile and silicone rubber relaxation curve obtained from the DMA (the acceleration calculation methods are given in the legend of each figure).

Table 4 Identified viscoelastic parameters using the nonlinear VFM with the one-term Ogden and Prony series model on the dropweight experiments on the nitrile and silicone rubber.

\begin{tabular}{|c|c|c|c|c|c|c|c|}
\hline Nitrile rubber & & & & & & & \\
\hline Temperature ${ }^{\circ} \mathrm{C}$ & $\mu_{0}(\mathrm{MPa})$ & $\alpha$ & $g_{1}$ & $g_{2}$ & $g_{3}$ & $g_{4}$ & $g_{5}$ \\
\hline 10 & 97.8 & 6.23 & 0.628 & 0.001 & 0.370 & 0.009 & 0.000 \\
\hline 14 & 103 & 0.83 & 0.711 & 0.001 & 0.236 & 0.058 & 0.000 \\
\hline 17 & 84.3 & 1.48 & 0.818 & 0.013 & 0.065 & 0.109 & 0.002 \\
\hline 21 & 55.2 & 0.47 & 0.795 & 0.018 & 0.054 & 0.103 & 0.019 \\
\hline 24 & 47.3 & 0.09 & 0.824 & 0.020 & 0.001 & 0.164 & 0.013 \\
\hline 28 & 31.1 & 1.04 & 0.799 & 0.002 & 0.001 & 0.208 & 0.009 \\
\hline 32 & 9.70 & 0.13 & 0.346 & 0.095 & 0.020 & 0.585 & 0.012 \\
\hline 39 & 5.19 & 0.22 & 0.086 & 0.010 & 0.006 & 0.970 & 0.005 \\
\hline 49 & 4.30 & 0.72 & 0.008 & 0.002 & 0.002 & 0.865 & 0.211 \\
\hline 54 & 3.65 & 0.21 & 0.000 & 0.000 & 0.000 & 0.319 & 0.842 \\
\hline 65 & 3.47 & 1.72 & 0.032 & 0.001 & 0.001 & 0.050 & 0.992 \\
\hline
\end{tabular}

\begin{tabular}{|c|c|c|c|c|c|c|c|}
\hline Silicone rubber & & & & & & & \\
\hline Temperature ${ }^{\circ} \mathrm{C}$ & $\mu_{0}(\mathrm{MPa})$ & $\alpha$ & $g_{1}$ & $g_{2}$ & $g_{3}$ & $g_{4}$ & $g_{5}$ \\
\hline 9 & 3.71 & 2.29 & 0.385 & 0.147 & 0.053 & 0.037 & 0.455 \\
\hline 14 & 2.72 & 3.44 & 0.273 & 0.112 & 0.046 & 0.230 & 0.234 \\
\hline 20 & 3.04 & 0.09 & 0.354 & 0.089 & 0.109 & 0.135 & 0.123 \\
\hline 22 & 2.38 & 3.32 & 0.230 & 0.111 & 0.181 & 0.063 & 0.096 \\
\hline 30 & 2.82 & 6.03 & 0.276 & 0.175 & 0.200 & 0.044 & 0.052 \\
\hline 41 & 2.77 & 2.79 & 0.447 & 0.105 & 0.034 & 0.007 & 0.365 \\
\hline
\end{tabular}




\begin{tabular}{llllllll}
49 & 1.70 & 2.75 & 0.189 & 0.065 & 0.119 & 0.028 & 0.359 \\
60 & 1.70 & 2.02 & 0.242 & 0.093 & 0.036 & 0.067 & 0.283 \\
\hline
\end{tabular}

\section{CONCLUSIONS}

This paper introduces the application of the nonlinear VFM for the identification of viscoelastic behaviour of rubbers. The identification aim is to characterize the local relaxation behaviour of rubber during dynamic deformation in a drop-weight experiment. The time-scale of the local relaxation was defined by the imaging speed and the period of each multiple loading period. The one-term Ogden model was adopted in the PVW of the nonlinear VFM and modified to include the viscoelastic model described by a Prony series model. This nonlinear VFM was validated by simulation works using the relaxation behaviours obtained from nitrile and silicone rubbers. In order to extend the period of the identified relaxation curve, the time-temperature superposition principle was utilized. Drop-weight experiments were conducted at several different temperatures. The local relaxation curves identified at each temperature were shifted with respect to the reference one measured at room temperature. The extended relaxation curve as a result of the shifting are well matched with the part of the relaxation behaviour measured from the DMA test for both of the nitrile and silicone rubber cases.

The lower limit of the time scale can be further extended if a higher imaging speed is adopted. However, this requires careful consideration because increasing the imaging speed gives a higher amplitude of spatial noise. For example, if $250000 \mathrm{fps}$ is adopted, the lower limit increases to -5.4 from -4.7 in terms of the logarithmic scale $(\log 10(\mathrm{~s}))$ but the noise amplitude on the axial displacement can become about 20 times higher (Yoon et al., 2015a). Instead, the application of lower temperatures is shown to be effective. At the moment, the present temperature device is only able to cool down to $9{ }^{\circ} \mathrm{C}$. It is expected that if a lower temperature can be applied, the identified relaxation curve can be further extended. For the extension of the upper limit of the time scale, a higher temperature range can be applied.

The present method has several advantages over the DMA test for particular cases. First, the use of the VFM allows the application of a proper constitutive model for rubbers so that the relaxation behaviour can be characterized with the consideration of a stress-strain nonlinearity. Second, a large specimen can be tested using the current method as the inertial effect is already counted in the VFM calculation. This advantage can be useful for composite or bio materials which needs to be large enough to fully exhibit their mechanical response. Third, whilst the model materials used here have relatively simple relaxation spectra, the method can be applied to materials exhibiting multiple relaxations or phase transitions, for which time-temperature superposition based on low frequency DMA data would be difficult to use. Here, the method presented in the current paper gives the opportunity to probe the material behaviour at frequencies similar to the room temperature transition frequency, and then use time-temperature superposition around this point.

Furthermore, the method can be extended to study the relaxation behaviour for larger deformations using the pre-stretching framework introduced in (Yoon et al., 2015a). The application of pre-stretching can be a useful study for rubbers exhibiting a nonlinear viscoelastic behaviour in which the relaxation is strain-dependent. Hence, the technique can give a range of data which currently cannot be obtained from either DMA or Hopkinson bar techniques. 


\section{ACKNOWLEDGEMENTS}

Effort sponsored by the Air Force Office of Scientific Research, Air Force Material Command, USAF, under grant number FA8655-12-1-2015. The U.S Government is authorized to reproduce and distribute reprints for Governmental purpose notwithstanding any copyright notation thereon. The authors thank S Fuller of AFOSR and M Snyder and R Pollak of EOARD for their support. The authors would like to thank R Froud, R Duffin and A Bateman for the construction of the experimental apparatus used in this research, and their helpful advice when designing this apparatus. Finally, we thank Professor F Pierron for his invaluable help with the Virtual Fields Method.

\section{REFERENCES}

ABAQUS, 2011. ABAQUS 6.11 analysis user's manual. Abaqus 6.11 Doc.

Bacon, C., 1998. An Experimental Method for considering Dispersion and Attenuation in a Viscoelastic Hopkinson Bar. Exp. Mech. 38, 242-249. doi:10.1177/001448519803800402

Bergström, J.S., Boyce, M.C., 1998. Constitutive modeling of the large strain time-dependent behavior of elastomers. J. Mech. Phys. Solids 46, 931-954. doi:http://dx.doi.org/10.1016/S0022-5096(97)00075-6

Chen, W., Lu, F., Zhou, B., 2000. A quartz-crystal-embedded split Hopkinson pressure bar for soft materials. Exp. Mech. 40, 1-6. doi:10.1007/BF02327540

Chen, W., Zhang, B., Forrestal, M.J., 1999. A split Hopkinson bar technique for low-impedance materials. Exp. Mech. doi:10.1007/BF02331109

Chen, W.W., 2016. Experimental Methods for Characterizing Dynamic Response of Soft Materials. J. Dyn. Behav. Mater. 2, 2-14. doi:10.1007/s40870-016-0047-5

Cheng, M., Chen, W., 2003. Experimental investigation of the stress-stretch behavior of EPDM rubber with loading rate effects. Int. J. Solids Struct. 40, 4749-4768. doi:10.1016/S00207683(03)00182-3

Connesson, N., Clayton, E.H., Bayly, P. V, Pierron, F., 2015. Extension of the optimised virtual fields method to estimate viscoelastic material parameters from 3D dynamic displacement fields. Strain 51, 110-134. doi:10.1111/str.12126

Gray, G.T., Blumenthal, W.R., 2000. Split-Hopkinson Pressure Bar Testing of Soft Materials. Mater. Park. OH ASM Int. 2000. 488-496.

Harding, J., Wood, E.O., Campbell, J.D., 1960. Tensile testing of materials at impact rates of strain. J. Mech. Eng. Sci. 2, 88-96. doi:10.1243/JMES_JOUR_1960_002_016_02

Harrigan, J.J., Ahonsi, B., Palamidi, E., Reid, S.R., 2014. Experimental and numerical investigations on the use of polymer Hopkinson pressure bars. Philos. Trans. A. Math. Phys. Eng. Sci. 372, 20130201. doi:10.1098/rsta.2013.0201

Kaliske, M., Rothert, H., 1997. Formulation and implementation of three-dimensional viscoelasticity at small and finite strains. Comput. Mech. 19, 228-239. doi:10.1007/s004660050171

Kanyanta, V., Ivankovic, A., 2010. Mechanical characterisation of polyurethane elastomer for biomedical applications. J. Mech. Behav. Biomed. Mater. 3, 51-62. doi:http://dx.doi.org/10.1016/j.jmbbm.2009.03.005

Kendall, M.J., Drodge, D.R., Froud, R.F., Siviour, C.R., 2014. Stress gage system for measuring very soft materials under high rates of deformation. Meas. Sci. Technol. 25, 075603. doi:10.1088/0957-0233/25/7/075603

Moulart, R., Pierron, F., Hallett, S., Wisnom, M., 2011. Full-Field Strain Measurement and Identification of Composites Moduli at High Strain Rate with the Virtual Fields Method. Exp. 
Mech. 51, 509-536. doi:10.1007/s11340-010-9433-4

Niemczura, J., Ravi-Chandar, K., 2011. On the response of rubbers at high strain rates-I. Simple waves. J. Mech. Phys. Solids 59, 423-441. doi:10.1016/j.jmps.2010.09.006

Ogden, R.W., 1972. Large Deformation Isotropic Elasticity - On the Correlation of Theory and Experiment for Incompressible Rubberlike Solids. Proc. R. Soc. London. A. Math. Phys. Sci. 326, 565-584. doi:10.1098/rspa.1972.0026

Pierron, F., Grédiac, M., 2012. The virtual fields method: extracting constitutive mechanical parameters from full-field deformation measurements. Springer, New York.

Pierron, F., Zhu, H., Siviour, C., 2014. Beyond Hopkinson's bar. Philos. Trans. R. Soc. A Math. Phys. Eng. Sci. 372, 20130195. doi:10.1098/rsta.2013.0195

Promma, N., Raka, B., Grédiac, M., Toussaint, E., Le Cam, J.B., Balandraud, X., Hild, F., 2009. Application of the virtual fields method to mechanical characterization of elastomeric $\begin{array}{llllll}\text { materials. } & \text { Int. } & \text { J. } & \text { Solids } & \text { Struct. } & \text { 46, }\end{array}$ doi:http://dx.doi.org/10.1016/j.jisolstr.2008.09.025

Roland, C.M., Twigg, J.N., Vu, Y., Mott, P.H., 2007. High strain rate mechanical behavior of polyurea. Polymer (Guildf). 48, 574-578. doi:10.1016/j.polymer.2006.11.051

Sasso, M., Chiappini, G., Rossi, M., Cortese, L., Mancini, E., 2013. Visco-Hyper-Pseudo-Elastic Characterization of a Fluoro-Silicone Rubber. Exp. Mech. 54, 315-328. doi:10.1007/s11340013-9807-5

Sihn, S., Tsai, S., 1999. Automated shift for time-temperature superposition. 12th Int. Com. Compos. Mater. 51.

Simo, J.C., 1987. On a fully three-dimensional finite-strain viscoelastic damage model: Formulation and computational aspects. Comput. Methods Appl. Mech. Eng. 60, 153-173. doi:10.1016/0045-7825(87)90107-1

Siviour, C.R., Jordan, J.L., 2016. High Strain Rate Mechanics of Polymers: A Review. J. Dyn. Behav. Mater. 2, 15-32. doi:10.1007/s40870-016-0052-8

Song, B., Chen, W., 2003. One-Dimensional Dynamic Compressive Behavior of EPDM Rubber. J. Eng. Mater. Technol. 125, 294. doi:10.1115/1.1584492

Williams, M., Landel, R., Ferry, J., 1955. The temperature dependence of relaxation mechanisms in amorphous polymers and other glass-forming liquids. J. Am. Chem. Soc. 679. doi:10.1021/ja01619a008

Yoon, S., Giannakopoulos, I., Siviour, C.R., 2015a. Application of the Virtual Fields Method to the uniaxial behavior of rubbers at medium strain rates. Int. J. Solids Struct. 1-16. doi:10.1016/j.ijsolstr.2015.04.017

Yoon, S., Winters, M., Siviour, C.R., 2015b. High Strain-Rate Tensile Characterization of EPDM Rubber Using Non-equilibrium Loading and the Virtual Fields Method. Exp. Mech. doi:10.1007/s11340-015-0068-3 